\title{
Non-organic stridor in children
}

\author{
Michael J. LaRouere and Charles F. Koop aann, Jr. \\ Depart: ont of Otolaryngology, University of Michigan, Ann Arbor, MI $48109-0312$ (U.S.A.) \\ (Received 18 March 1987) \\ (Revised version received 26 August 1987) \\ (Accepted 31 August 1987)
}

Key words: Non-organic; Stridor; Child

\section{Summary}

A 4.75-year-old male presented with several episodes of expiratory stridor leading, on one occasion, to a respiratory arrest and intubation. A detailed evaluation emphasizing physiological and anatomical studies revealed no organic basis for the patient's upper airway obstruction. Emphasis is placed on one method found to be helpful in the differentiation of an organic versus a psychogenic type of respiratory disorder. Recognition of functional airway obstruction in children may prevent inappropriate therapy and allow proper psychiatric intervention.

\section{Introduction}

Rogers and Stell [13] in 1978 ïist reported two cases of functional inspiratory stridor caused by paradoxical epproximation of the vocal cords. Since this report there have been sporadic case presentations of non-organic inspiratory stridor $[1,3,4,7,8,12]$. These studies were in agreement with Rogers and Stell's observation that paradoxical movement of the vocal cords (adduction) during inspiration was responsible for the stridor. The majority of these patients were female and had some form of psychiatric illness $[1,3,5,7,13]$. Several of these patients presented with acute upper respiratory tract obstruction requiring intubation or tracheotomy $[1,3,5,8]$. No organic cause for paradoxical vocal cord movement could be found. Stanescu reported the first case of functional expiratory st idor in 1982, coining the term emotional laryngeal wheezing [14]. Other terms, iach as fictitious asthma [6], have been used to describe this entity.

Correspondence: C.F. Koopmann, Department of Otolaryngology, University of Michigan, 15(? East Medici:l Center Drive, Box 0312, Ann Arbor, MI 48109-0312, U.S.A. 
We present the case of a patient whose sudden attacks of respiratory stridor led to the diagnosis of croup and asthma. A thorough diagnostic evaluation has enabled us to exclude an organic cause for this patient's stridor and to demonstrate one method found to be useful in the differentiation of an organic versus a psychogenic type of respiratory disorder.

\section{Case report}

A 4.75-year-old male presented to another hospital in acute respiratory distress (cyanosis, tachycardia to 120 , stridor, and severe sternal retractions). He was not wheezing and was afebrile. After failing to respond to an initial treatment of oxygen, epinephrine and i.v. aminophylline, he was thought to have a respiratory arrest which resulted in the administration of mouth to mouth resuscitation. After 5 breaths the patient resumed breathing spontaneously, but was subsequently intubated. At the time of intubation he was thought to have an enlarged, inflamed epiglottis. Arterial blood gases on $100 \%$ oxygen revealed a pH of 7.31, a $p \mathrm{CO}_{2}$ of 39 , and a $\mathrm{pO}_{2}$ of 394 with a bicarbonate level of 19. A chest roentgenogranı was normal. He was then transferred to Mott Children's Hospital (MCH) at the University of Michigan.

Upon arrival at $\mathrm{MCH}$ the patient was placed on a respirator because muscle relaxants had been given en route. A chest roentgenogram was normal. Arterial blood gasses on $40 \%$ nxygen showed a pH of 7.36, a $p \mathrm{CO}_{2}$ of 40 , and a $\mathrm{pO}_{2}$ of 94 . The epiglottis was normal on direct laryngoscopy. Consequently the patient was extubated $2 \mathrm{~h}$ after admission without showing signs of wheezing or stridor. Arterial blood gasses after extubation showed a $\mathrm{pH}$ of 7.37, a $p \mathrm{CO}_{2}$ of 42 and a $p \mathrm{O}_{2}$ of 91 .

The past medical history revealed that over the previous 24 months the patient had at least 6 similar episodes of acute respiratory distress. Each bout was characterized by sudden expiratory stridor without an antecedent upper respiratory tract infection or wheezing. The attacks would last from several minutes to over an hour and were unresponsive to bronchodilator therapy. Three of these episodes required evaluation in the emergency room. Two attacks resolved without treatment while the third resolved during the administration of epinephrine and aminophylline. Skin testing was negative and there was no family history of atopy or allergy.

After extubation the patient had no stridor. AP and laterai soft tissue roentgenograms of the neck and a repeat chest roentgenogram revealed no abnormalities. An upper $\mathrm{Gl} /$ barium swallow was normal. Blood and sputum cultures revealed no growth. Pertussis titers were negative. $C_{1}$ esterase inhibitor, $C_{2}$ and $C_{4}$ levels, as well as maximum inspiratory and expiratory flow volume relationships were normal. These flow volume measuremeats occurred while the patient was symptom free. Methacholine chloride inhalation challenge (Table I) was also within the normal range (bronchial hyper-reactivity being defined as a reduction in the forcod expiratory volume of $20 \%$ or more in cae second as compared to an initial baseline) [10].

The patient underwent direct laryngoscopy and bronchoscopy under general anesthesia. No abnormalities were noted. Several minutes after an uneventful 


\section{TABLE I}

FVC tests pre- and post-methacholine challenge

Trial 1 = AVG of two trials; trial 2 = trial after challenge with $25 \mathrm{mg} / \mathrm{ml}$ of methacholine. No significant difference noted between trials. FVC, forced vital capacity musasured in liters; FEV1, forced expiratory volume at $1 \mathrm{~s}$; F25-75, average flow between 25\%-75\% of FVC (forced vital capacity); PF, peak flow expressed in 1/s; MEF-50, maximum expiratory flow which is $50 \%$ of forced vital capacity (FVC); IVC, inspiratory vital capacity expressed in liters.

\begin{tabular}{lllllll}
\hline Trial & FVC & FEVI & F25-75 & PF & MEF-50 & IVC \\
\hline 1 & 0.83 & 0.72 & 0.90 & 2.14 & 1.00 & 0.84 \\
2 & 0.85 & 0.78 & 1.04 & 2.26 & 1.16 & 0.40 \\
\hline
\end{tabular}

extubation, the patient developed expiratory stridor localized to the upper airway which transmitted to an otherwise clear lung field. Under controlled conditions in the operating room, $30 \mu \mathrm{g}$ of Fentanyl were slowly administered i.v. with resolution of the stridor. Upon arousal the patient had no further respiratory distress.

Post-operative intervention included a psychological assessment which revealed marked dependence of the patient on his mother. The pcssibility of night terrors or anxiety related respiratory distress was entertained. He appeared to meet criteria for an adjustment disorder with mixed emotional features. Speech therapy evaiuation revealed a moderate to marked speech and stuttering problem which warrarted formal therapy.

\section{Discussion}

The differential diagnosis of airway obstruction in children includes infection (croup and acute epiglottitis); neoplasms; foreign bodies; allergic reactions; angioneurotic edema; trauma; typical and atypical pertussis; congenital airway abnormalities including: laryngomalacia, iaryngeal webs, vocal cord paralysis, subglottic stenosis, subglottic hemangioma, and compression from anomalous great vessels [11]. Narrowing of the tracheobronchial tree causing expiratory stridor can also be the result of asthma [9]. To this differential, functional laryngospasm and bronchospasm can be added.

This patient's presentation contained several disciepancies. For two years the sudden attacks of expiratory stridor (lasting minutes to over an hour) failed to respond to the usual treatment for bronchial asthma; however, a diagnosis of asthma and recurrent croup had been made. Critical evaluation of his history disclosed features that were inconsistent with these diagnoses. The attacks occurred suddenly, without any antecedent airv ay abnormality such as an elevated temperature, hoarseness, wheezing pior to or between an event, or evidence of upper respiratory tract infection precipitating croup. Not onily was there a lack of wheezing on chest auscultation, but bronchodilators failed to alleviate the obstruc- 
tion. Evaluation of the patient's laboratory data on admission shows an acidosis consistent with asthma or airway obstruction, althwugh the carbon dioxide level was normal. Further, his initial and subsequent chest roentgenograms did not reveal hyperinflation as is usually seen in severe asthmatic attacks.

The inconsistencies in this patient's presentation represented a unique diagnostic challenge. The atypical features and his markedly withdrawn personality led us to consider functional airway obstruction as a possible diagnosis prior to endoscopy.

At the conclusion of an uneventfui (where no lesions were identified) endoscopy, our patient was easily extubated without respiratory difficulty. However, when more alert, he exhibited marked expiratory stridor. Under controlled conditions in the operating room, sedating the patient with $30 \mu \mathrm{g}$ of Fentanyl quickly resolved the stridor and on arousal these was no evidence of further airway obstruction. It has long been a dictum that tranquilizers or agents which cause respiratory depression should not be used in acutely ill asthmatics for fear of respiratory depression and precipitation of a respiratory arrest [9]. Conversely, sedation in the presence of a disorder believed to be functional, as in adductor cord spasm causing inspiratory or expiratory stridor, may relieve the obstruction $[5,8]$. Thus, under optintal conditions, after a thorough historical, physiological and anatomical evaluation, sesation during an acute episode of expiratory stridor may be helpful differentiating a functional from an organic cause of airway obstruction.

Respiratory distress secondary to functional expiratory stridor appears closely associated with functional inspiratory stridor caused by paradoxical vocal cord motion. The majority of the latter group of patients are females who exhibit psychological disorders and in whom careful evaluation has failed to reveal an organic cause for the episodes of vocal cord adduction during inspiration. Many of these patients are helped with tranquilizers [5,8]. The two disorders may be a means for the psychologically disturbed patient to manipulate their environment. Establishing the diagnosis of non-organic disease is very important to the patient, the family and to the involved health care providers. A thorough objective evaluation emphasizing physiological tests and anatomical studies is then nerded to exclude organic pathology prior to establishing a functional diagnosis.

Patients who do not have the more common airway anomalies, which can usually be documented endoscopically and radiographically, should be evaluated for systemic problems. Normal $C_{1}$ esterase initibitor, $C_{2}, C_{4}$ and antipertussis fluorescent antibody levels can exclude angioneurotic edema and pertussis. The majority of asthmatics will have inducible bronchial hyperreactivity and methacholine challenge has been demonstrated to be the best method to evaluate this [10].

In conclusion, functional expiratory stridor has rarely been reported and appears very similar to asthma [9] or spasmodic croup [2]. However, in the proper historical setting and with a detailed evaluation, including sedation of the patient under controlled conditions, recognition of this clinical entity may prevent inappropriate therapy, avold unnesessary intubations and ailow appropriate psychiatric intervention. 


\section{References}

1 Appelbiat:, N.E. and Dake- SR, Functisn? wper airvay ohstruction: a new syndrome, Arch. Otolaryngol., 107 (1981) 305-306.

2 Cherry, J.D., The treatment of croup: continued controversy due to failure of recognition of historic, ecologic, etiologic and clinical perspectives, J. Pediatr., 94 (1979) 352-354.

3 Christopher, K.L., Wood, R.P., Eckert, R.C., Blager, F.B., Raney, R.A. and Souhrada, J.F., Vocal cord dysfunction presenting as asthma, N. Engl. J. Med., 308 (1983) 1566-1570.

4 Collett, P.W., Brancatisano, T. and Engel, L.A., Spasmodic croup in the ajult, Am. Rev. Respir. Dis., 127 (1983) 500-504.

5 Cormier, Y.F., Camus, P and Desmeules, M.J., Non-organic acute upper airway obstruction: deseription and a diagnostic approach, Am. Rev. Resp. Dis., 121 (1980) 147-150.

6 Downing. E.T., Braman, S.S., Fox, M.J. and Corrao, W.M., Factitious asthma: physiological approach to diagnosis, J. Am. Med. Ass., 248 (1982) 2878- 2881.

7 Kattan, M. and Ben-Zvi, Z., Stridor caused by vocal cord malfunction associated with emotional factors, Clin. Ped., 24 (1985) 158-160.

8 Kellman, R.M. and Leopold, D.A., Paradoxical vocal cord motion: an important cause of stridor, Laryngoscope, 92 (1982) 58-60.

9 McFadden, E.R. and Austen, K.F., Lung Disease Caused by Immunologic and Environmental Injury. Harrison's Principles of Internal Medicine. 9th edn. McGraw-Hill, New Y'ork, 1980, pp. 1203-1210.

10 Parker, C.D., Bilbo, R.E. and Reed C.F., Methacholine aerosol as a test for bronchial asthma, Arch. Int. Med., 115 (1965) 452-458.

11 Pattersion, R.. Schatz, M. and Horton, M., Munchausen's stridor: non-organic laryngeal obstruction, Clin. Allergy, 4 (1974) 307-310.

12 F ogers, J.H.. Functional inspiratory strider in children, J. Laryngol. Otol., 94 (1980) 669-670.

13 Mogers, J.H. and Stell, F.M., Paradoxical movement of the rocal cords as a cause of stridor, I. Laryigol. Otol., 32 (1978) 157-158.

14 Stanescu, D.C., Francis, C. and Rodenstein, D.O., Emotional lar yngeal wheezing: a new syndrome, Añ. Rev. Respir. Dis., 1.27 (1983) 354-356. 